\title{
Survey on Dual Sentiment Analysis
}

\author{
Akshay Uttarwar ${ }^{1}$, Akash Ithape ${ }^{1}$, Rajat Pawar ${ }^{1}$, Rushikesh Jaybhay ${ }^{1}$, Prof. Vaishali Baviskar ${ }^{2}$ \\ Bachelors in Engineering, Computer, G.H Raisoni Institute of Engineering and Technology, Pune, India ${ }^{1}$ \\ Professor, Computer, G.H Raisoni Institute of Engineering and Technology, Pune, India ${ }^{2}$
}

\begin{abstract}
Mining opinion targets and opinion words from online reviews of products (Custom portal) are important tasks for fine-grained opinion mining, the key component of which involves detecting opinion relations among words (target words). In this system user can add their reviews about the product and the system can extract the target words and opinion relation. By using the opinion and targets to find out the sentiment analysis about the reviews. Reviews contains the compound sentence then it will split it into the separate sentence and find the targets and opinion and also sentiment from reviews. The system is then reversing the Negative opinion sentences into Positive. The sentiment can be negative, positive, neutral, vary negative and very positive. Then all the sentiments, targets and the opinion about the product are represented using the graphical format. All comments will be stored in Hadoop and Analysis will be done on comments using Hadoop.
\end{abstract}

Keywords: Hadoop, Data Mining, Sentiment Analysis.

\section{INTRODUCTION}

In recent years, with the growing volume of online reviews available on the Internet, sentiment analysis and opinion mining, as a special text mining task for determining the subjective attitude (i.e., sentiment) expressed by the text, is becoming a hotspot in the field of data mining and natural language processing .Sentiment classification is a basic task in sentiment analysis, with its aim to classify the sentiment (e.g., positive or negative) of a given text. The general practice in sentiment classification follows the techniques in traditional topicbased text classification, where the Bag-of-words (BOW) model is typically used for text representation. In the BOW model, a review text is represented by a vector of independent words. By using natural language processing techniques become easy to analysis sentence in positive and negative class from this we can using through sentiment analysis is possible to recognize positive and negative sentences and can convert that sentences into positive manner e.g.: if some user take product and give comment on that product is negative like. "he didn't like the product "so this application is made for product growth purpose so this application convert his comment on positive manner and shows other user in positive like "he like the product" from this application product will be take profit never get loss from users comment.

\section{REVIEW OF RELATED LITERATURE}

In survey we observed user comment is very important for other user who's going to buy the product if already bought user give negative comment on product then new user cannot want to buy such product so the product growth going to down and product company also getting loss so giving attention on this problem we are developing dual sentiment analysis system which is analysis user comment if user comment coming in negative so this system convert this comment into positive comment and generate graph and based on the score it [2] show graph and from this owner of product also getting review of product so from this system give help to owner to make product with users requirement and make it good. In this we extend our previous work in three major aspects. First, we strengthen the DSA algorithm by adding a selective data expansion procedure. Second, we extend the DSA framework from sentiment polarity classification to positive-negative-neutral sentiment classification. Third, we propose a corpus-based method to construct a pseudoantonym dictionary that could remove DSA's dependency on an external antonym dictionary.

\section{RESERCH METHODOLOGY}

According to the levels of granularity, tasks in sentiment analysis can be divide into four categorizations: document level, sentence-level, phrase-level, and aspect-level sentiment analysis. Focusing on the phrase/sub sentence and aspect-level sentiment analysis, Wilson et al. [42] discussed effects of complex polarity shift. They began with a lexicon of words with established prior polarities, and identify the "contextual polarity" of phrases, based on some refined annotations.

Choi and Cardie [4] further combined different kinds of negators with lexical polarity items though various compositional semantic models, both heuristic and machine learned, to improved sub sentential sentiment analysis. Nakagawa et al. [29] developed a semi supervised model for sub sentential sentiment analysis that predicts polarity based on the interactions between nodes in dependency graphs, which potentially can induce the scope of negation. In aspect-level sentiment analysis, the polarity shift problem was considered in both corpus- and lexicon-based methods. 
Vol. 5, Issue 12, December 2016

For document- and sentence-level sentiment classification, and give it to the sentiment analysis. Sentence reversion there are two main types of methods in the literature: term- e.g. 'I don't like product' will be reverse as 'I like counting and machine learning methods. In term-counting product'. Means Negation removal. Generate the graph methods, the overall orientation of a text is obtained by based on to the sentiment and its score. User having the summing up the orientation scores of content words in the authority to delete the own comment. So this system give text, based on manually-collected or external lexical all this feature to make profit of product.

resources [38], [39]. In machine learning methods, sentiment classification is regarded as a statistical classification problem, where a text is represented by a bag-of words; then, the supervised machine learning algorithms are applied as classifier [35]. Accordingly, the way to handle polarity shift also differs in the two types of methods.

\section{A. Issues and Challenges}

Followings are the issues an challenges which are faced to implement in India-police system.

1) Inadequate ICT Infrastructure across the nation.

2) Inadequate access to ICT by citizen.

3) Lack of awareness of police personnel an citizens about ICT.

4) Lack of adequate training programs in India

5) Inadequate ICT-trained people.

6) Lack of reliable maintenance an sustainability.

7) Lack of mother language standardization.

8) Insufficient of electricity across the nation.

9) High-cost an low-reliability of Internet access.

10)Lack of awareness of future development.

\section{IV.SYSTEM ARCHITECTURE}

\section{A. Basic Architecture}

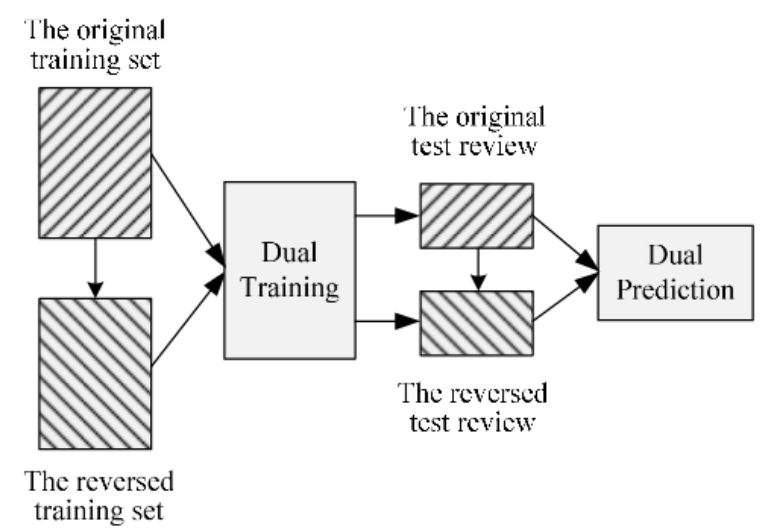

Fig. 1: The process of dual sentiment analysis. The rectangle filled with slash denotes the original data, and the rectangle filled with backslash denotes the reversed data.

Fig. 1Architecture diagram for application

In proposed system we are develop dual sentiment analysis system. In this we taking two users in this system admin and multiple user/ customer. Admin can add the Products and Product category having the authority to delete the product and Product category.

User gives comment to particular product. On comment text Apply NLP on the comment text then extract the opinion and targets from comment using the Stanford NLP

\section{B. Activity diagram}

Activity diagram is another important diagram in UML to describe dynamic aspects of the system. Activity diagram is basically a flow chart to represent the flow form one activity to another activity. The activity can be described as an operation of the system.

Fig to show the activity diagram which represent the multiple activity to be undertaken by the admin and the surveyor to produce the graph based structure for effective decision making. The activity diagram also indicate action to be taken in event of captured of a suspect.

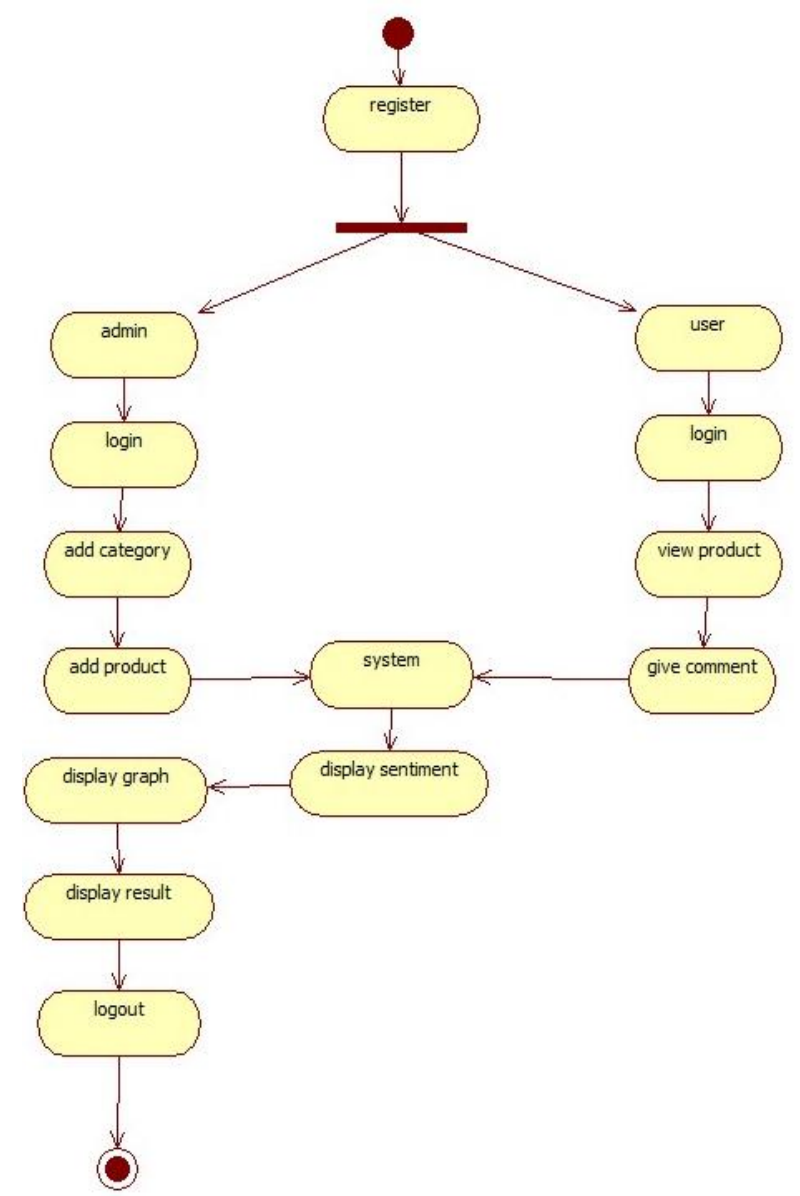

Fig.2. Activity Diagram

\section{DISCUSSION}

This system can convert the negative comment on product into the positive comment. Also it generate the graph representation for each sentiment, Which useful to reduce the user overhead i.e to read all reviews user can easily analyze using graphs and make a decision. 


\section{CONCLUSION}

From we can conclude this system giving help to owner of product to make the product quality changes as per user requirement from this system graph of user comment and this system convert all negative comment of user into positive comment of user. To make help for growth of product.

\section{ACKNOWLEDGMENT}

We thank our colleagues, who provided insight and expertise that greatly assisted the research. We thank our Prof. Vaishali Baviskar for inspiring us to do this project and her comments on the manuscript.

\section{REFERENCES}

[1] Abbasi, S. France, Z. Zhang, and H. Chen, "Selecting attributes for sentiment classification using feature relation networks," IEEE Transactions on Knowledge and Data Engineering (TKDE), vol. 23, pp.447

[2] E. Agirre, and D. Martinez, "Exploring automatic word sense disambiguation with decision lists and the Web," Proceedings of the COLING Workshop on Semantic Annotation and Intelligent Content, pp. 11-19,

[3] J. Cano, J. Perez-Cortes, J. Arlandis, and R. Llobet, "Training set expansion in handwritten character recognition," Structural, Syntactic, and Statistical Pattern Recognition, pp. 548-556, 2002. 